\title{
Determination of the effective sample thickness via radiative capture
}

\author{
A. M. Hurst ${ }^{\mathrm{a}, *}$, N. C. Summers ${ }^{\mathrm{b}}$, L. Szentmiklósic ${ }^{\mathrm{c}}$, R. B. Firestone ${ }^{\mathrm{a}}$, M. S. Basunia ${ }^{\mathrm{a}}$, J. E. Escher $^{\mathrm{b}}$, B. W. Sleaford ${ }^{\mathrm{b}}$ \\ ${ }^{a}$ Lawrence Berkeley National Laboratory, Berkeley, California 94720, USA \\ ${ }^{b}$ Lawrence Livermore National Laboratory, Livermore, California 94550, USA \\ ${ }^{c}$ Centre for Energy Research, Hungarian Academy of Sciences, H-1525 Budapest, Hungary
}

\begin{abstract}
A procedure for determining the effective thickness of non-uniform irregular-shaped samples via radiative capture is described. In this technique, partial $\gamma$-ray production cross sections of a compound nucleus produced in a neutron-capture reaction are measured using Prompt Gamma Activation Analysis and compared to their corresponding standardized absolute values. For the low-energy transitions, the measured cross sections are lower than their standard values due to significant photoelectric absorption of the $\gamma$ rays within the bulk-sample volume itself. Using standard theoretical techniques, the amount of $\gamma$-ray self absorption and neutron self shielding can then be calculated by iteratively varying the sample thickness until the observed cross sections converge with the known standards. The overall attenuation, thus, provides a measure of the effective sample thickness illuminated by the neutron beam. This procedure is illustrated through radiative neutron capture using powdered oxide samples comprising enriched ${ }^{186} \mathrm{~W}$ and ${ }^{182} \mathrm{~W}$ from which their tungsten-equivalent effective thicknesses are deduced to be $0.077(3) \mathrm{mm}$ and $0.042(8) \mathrm{mm}$, respectively.
\end{abstract}

Keywords: Prompt Gamma Activation Analysis (PGAA), $\gamma$-ray absorption, neutron attenuation, $(n, \gamma)$, radiative capture, partial $\gamma$-ray production cross sections, effective sample thickness.

\section{Introduction}

For accurate nuclear-data measurements, targets with welldefined geometries, thicknesses, homogeneities and compositions (e.g. thin metal foils and pellets) are preferred. In many cases, however, this ideal case cannot be achieved and there are several applications of in-beam measurements where the irradiated sample may take an irregular inhomogeneous form. For irradiated powders in particular, the material will need to be held in a sample holder such as a Teflon bag. Consequently, the sample mass is unlikely to distribute itself into a regular shape with a uniform surface; measuring the areal density and thickness, or the average pile density, of these non-uniform samples then becomes extremely challenging. For these samples, accurately quantifying the induced $(n, \gamma)$ reaction rate, for example, from deexcitation via $\gamma$-ray emission, may become complicated. This is especially true for high-density materials with low-to-moderate neutron-absorption cross sections, for example, tungsten, since neutron self-shielding and $\gamma$-ray absorption within the sample itself is significant and large corrections are needed. Regular geometries may be modeled rather well using particle-transport simulation toolkits such as MCNP6 [1] and GEANT4 [2]. However, insufficient knowledge of the geometric distribution of the sample, i.e. its thickness, makes it difficult to correct for attenuation in a robust and reliable manner and so alternative procedures must be developed. Some examples of methodologies that have previously been adopted to address this problem include: (i) Measuring neutron-fluence rates

\footnotetext{
Corresponding author

Email address: AMHurst@lbl.gov (A. M. Hurst)
}

in Prompt Gamma Activation Analysis (PGAA), where the irradiated sample is held in and out of beam, to determine relative self-shielding factors and establish the average pile density of the sample [3]; (ii) Comparing full-energy photopeaks from ideally-thin calibration standards to the same transitions from thick samples to establish the $\gamma$-ray energy-dependent $\left(E_{\gamma}\right)$ correction factors for neutron self shielding and photoattenuation [4]; (iii) Semi-quantitative Monte Carlo simulations to reproduce measured $\gamma$-ray intensity maps by iteratively adjusting neutron self absorption and photoattenuation correction factors to minimize residuals between measurement and simulation [5].

Indeed, if self-attenuation processes are not handled accurately, this can lead to misleading partial $\gamma$-ray production cross sections $\left(\sigma_{\gamma}\right)$ for samples measured using PGAA setups [6, 7]. This problem was highlighted recently in a study of the tungsten isotopes [8] where the new $\sigma_{\gamma}$ values were found to differ considerably from those in the current Evaluated Gamma-ray Activation File (EGAF) [9, 10] repository owing to neutronabsorption and $\gamma$-ray self-attenuation considerations. In this paper, we present a method for determining the effective thickness of a non-uniform sample via radiative capture through exposure to a flux of near-thermal neutrons. Here, we define the effective thickness as the mean thickness seen by the neutrons and $\gamma$ rays in an equivalent planar geometry corresponding to a homogeneous sample of the same material. As a validation of the present methodology, we also compare our results to those obtained using a suitably-thin reference calibration standard, similar to the procedure outlined in Ref. [4]. 


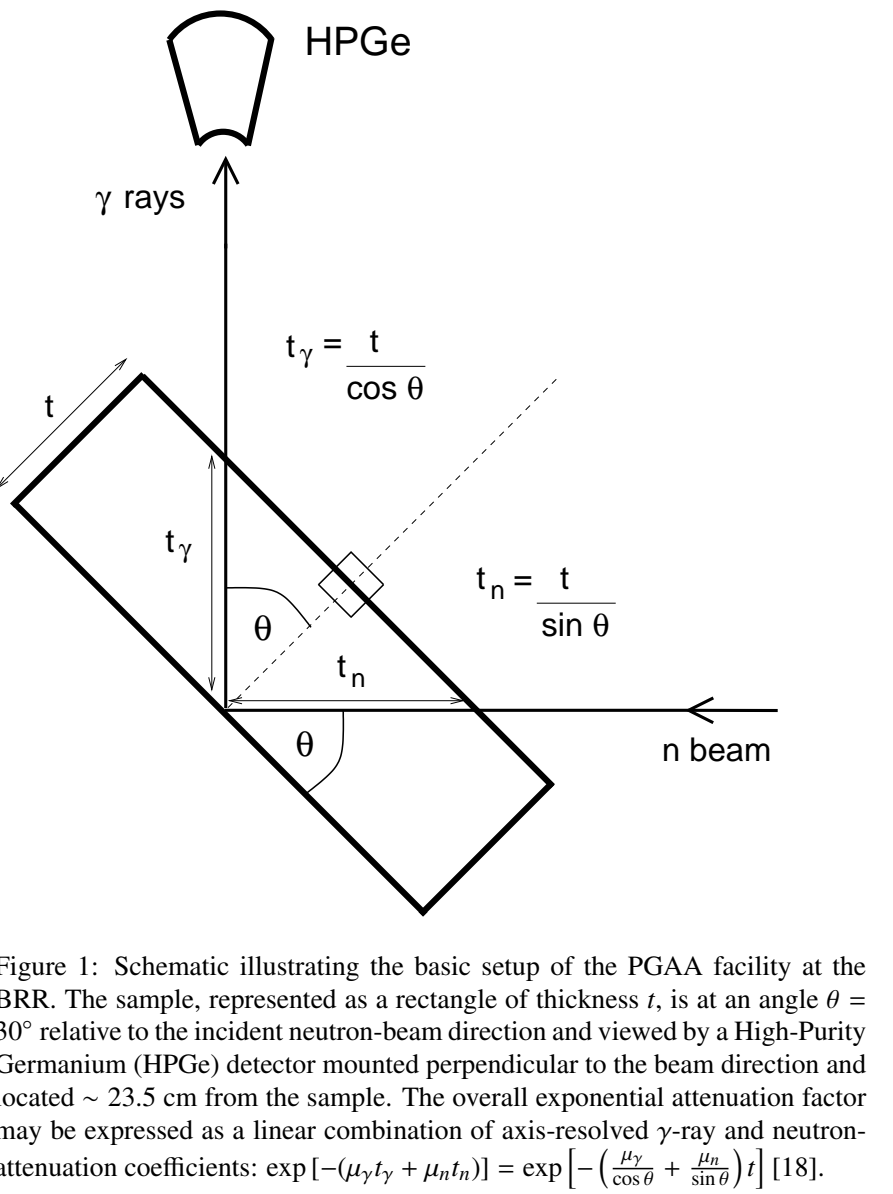

\section{Method}

A series of near-thermal $(T=120 \mathrm{~K})$ neutron-capture measurements using isotopically-enriched and natural samples of tungsten oxide $\left(\mathrm{WO}_{2}\right)$ powders have recently been carried out [8] at the 10-MW Budapest Research Reactor (BRR) $[11,12,13,14,15,16]$. This work employed the PGAA setup $[6,7]$ to measure absolute neutron-capture partial $\gamma$-ray production cross sections in tungsten isotopes produced via the radiative $(n, \gamma)$ reaction with samples of enriched ${ }^{182,183,186} \mathrm{~W}$ and

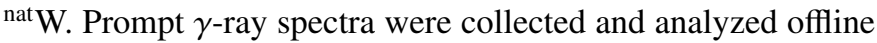
using the $\gamma$-ray spectroscopy software package HYPERMET-PC [17]. Full details of the experimental setup and corresponding results are published elsewhere [8]. To obtain these precise cross sections, however, requires accurate knowledge of the effective sample thickness exposed to the neutron beam during irradiation. Samples that are not ideally thin, such as the high-density tungsten samples $\left(\rho(\mathrm{W})=19.25 \mathrm{~g} / \mathrm{cm}^{3}\right.$, $\left.\rho\left(\mathrm{WO}_{2}\right)=10.8 \mathrm{~g} / \mathrm{cm}^{3}\right)$ used in this work, are not fully transparent to neutrons and $\gamma$ rays. Furthermore, because the distribution of the neutron beam is generally not spatially uniform, and in practice the beam aperture is often smaller than the actual volume of the bulk sample material, the irradiated sample may not be fully illuminated by the neutron beam. Additionally, the partial illumination zone of the sample may not be in full view of a collimated $\gamma$-ray detector. These considerations may, thus, contribute significantly to the effective sample thickness deduced from the measured peak areas in PGAA, appropriately corrected for neutron self shielding and $\gamma$-ray self absorption due to attenuation within the observed illumination zone of the sample itself.

In Sect. 2.1 and 2.2, respectively, we first outline the theoretical formalism governing the self-attenuation processes, and then describe how this information can be used to experimentally infer the effective thickness of the irradiated sample.

\subsection{Self-attenuation correction}

In general, scattered photons will emerge from an irradiated sample with a diminished photon intensity $I$ according to the exponential-attenuation law

$$
I=I_{0} \mathrm{e}^{-\mu x},
$$

where $I_{0}$ represents the true unattenuated photon intensity, $x$ is the sample thickness, and $\mu$ is a coefficient describing the linear combination of neutron- $\left(\mu_{n}\right)$ and $\gamma$-ray $\left(\mu_{\gamma}\right)$ attenuation coefficients for a given sample i.e. $\mu=\mu_{\gamma}+\mu_{n}$. The angle $\theta$ of the sample holder relative to the incident beam direction, depicted by the geometry in Fig. 1, must also be taken into account in determining the overall attenuation coefficient, thus

$$
\mu=\frac{\mu_{\gamma}}{\cos \theta}+\frac{\mu_{n}}{\sin \theta} .
$$

This angle has been measured to be $30^{\circ}$ at the BRR. The analytic form of the $\gamma$-ray energy-dependent attenuation factors [18] may then be obtained from the numerical integration of Eq. 1 over the sample thickness $t$

$$
\begin{aligned}
\frac{I}{I_{0}} & =\int_{x=0}^{x=t} e^{-\left(\frac{\mu_{\gamma}}{\cos \theta}+\frac{\mu_{n}}{\sin \theta}\right) x} \cdot d x \\
& =\frac{1}{\left(\frac{\mu_{\gamma}}{\cos \theta}+\frac{\mu_{n}}{\sin \theta}\right)} \cdot\left[1-e^{-\left(\frac{\mu_{\gamma}}{\cos \theta}+\frac{\mu_{n}}{\sin \theta}\right) t}\right] .
\end{aligned}
$$

In this work, we derived the $\gamma$-ray energy dependent $\mu_{\gamma}$ values from XMuDat [19], a database of mass-attenuation coefficients, $\mu_{\gamma} / \rho$, generated according to prescriptions outlined in Refs. [20, 21]. Thus, for a natural sample of mono-elemental composition $\mu_{\gamma}=\left(\mu_{\gamma} / \rho\right) \rho$, where $\rho$ is the sample density. For compounds, however, we must introduce a weighting factor $w_{i}$ to account for the constituent elements in the compound

$$
w_{i}=\frac{A_{i}}{M} \quad \text { where } \quad M=\sum_{i}^{j} A_{i} .
$$

Here, $A_{i}$ is atomic mass of element $i$ and $M$ is the total stoichiometric mass of the compound. The $\gamma$ attenuation coefficients for a compound sample may then be deduced using the expression

$$
\mu_{\gamma_{x}}=\left(\sum_{i}^{j} \frac{\mu_{\gamma_{i}}}{\rho_{i}} w_{i}\right) \rho_{x},
$$


Table 1: Transition energies $\left(E_{\gamma}\right)$ from ${ }^{186} \mathrm{~W}(n, \gamma){ }^{187} \mathrm{~W}$ and their corresponding absolute neutron-capture partial $\gamma$-ray production cross sections $\left(\sigma_{\gamma}^{\mathrm{S}}\right)$ obtained from a standardized $\mathrm{H}_{2} \mathrm{WO}_{4}(n, \gamma)$ measurement [8]. Standardization energies and cross sections from ${ }^{182} \mathrm{~W}(n, \gamma)^{183} \mathrm{~W}$ are also presented. The transitions are indexed (i) in order of ascending $E_{\gamma}$. The efficiency-corrected peak areas, denoted $A_{\gamma}^{\prime}$, were measured using enriched powdered samples of ${ }^{186} \mathrm{~W}$ (99.65(3)\%) and ${ }^{182} \mathrm{~W}$ (92.7(9)\%); $A_{\gamma}^{c}$ represent the same peak areas corrected for $\gamma$-ray absorption and neutron self shielding assuming the deduced effective sample thicknesses of $0.077 \mathrm{~mm}$ and $0.042 \mathrm{~mm}$, respectively. In the final column the proportionality constant, $R_{i}\left(E_{\gamma_{i}}\right)$, relating $\sigma_{\gamma}^{\mathrm{S}}$ and $A_{\gamma}^{c}$ is listed. See text for details.

\begin{tabular}{|c|c|c|c|c|c|c|}
\hline \hline Sample & $E_{\gamma}[\mathrm{keV}]$ & $i$ & $\sigma_{\gamma}^{\mathrm{S}}[\mathrm{b}]$ & $A_{\gamma}^{\prime}[\mathrm{cnt}]$ & $A_{\gamma}^{\mathrm{c}}[\mathrm{cnt}]$ & $R_{i}\left(E_{\gamma_{i}}\right)\left[\mathrm{b} \cdot \mathrm{cnt}^{-1}\right]$ \\
\hline${ }^{186} \mathrm{~W}$ & $77.30(5)$ & 1 & $0.234(4)$ & $13361(321)$ & $25950(623)$ & $9.02 \times 10^{-6}(27)$ \\
& $145.84(5)$ & 2 & $1.344(13)$ & $124860(5619)$ & $146941(6612)$ & $9.15 \times 10^{-6}(42)$ \\
& $273.12(5)$ & 3 & $0.380(4)$ & $38868(3848)$ & $41078(4066)$ & $9.25 \times 10^{-6}(92)$ \\
& $5261.67(9)$ & 4 & $0.653(9)$ & $70901(1205)$ & $72449(1231)$ & $9.01 \times 10^{-6}(20)$ \\
\hline \hline${ }^{182} \mathrm{~W}$ & $98.90(1)$ & 1 & $0.0906(32)$ & $11602(174)$ & $14375(215)$ & $6.30 \times 10^{-6}(24)$ \\
& $162.11(1)$ & 2 & $0.2605(72)$ & $37534(450)$ & $40356(483)$ & $6.45 \times 10^{-6}(19)$ \\
& $291.57(1)$ & 3 & $0.0665(25)$ & $10358(124)$ & $10644(127)$ & $6.25 \times 10^{-6}(25)$ \\
& $1100.39(3)$ & 4 & $0.0334(14)$ & $5282(137)$ & $5349(138)$ & $6.24 \times 10^{-6}(31)$ \\
& $6190.78(6)$ & 5 & $0.726(10)$ & $112700(1578)$ & $114043(1596)$ & $6.37 \times 10^{-6}(13)$ \\
\hline \hline
\end{tabular}

where $\rho_{x}$ is the measured density of the compound sample, and the summation is performed over all constituent elements $i$ in the compound. The authors of the XMuDat database claim a precision of $5 \%$ for the absorption data, so this was adopted as a conservative estimate to derive $\Delta_{\mu_{\gamma}}$, the uncertainty in $\mu_{\gamma}$. For thermal-neutron temperatures and lower, the neutronattenuation coefficients may be computed from the neutronabsorption cross sections $\sigma_{\text {nabs }}$ listed in Ref. [22] according to

$$
\mu_{n_{x}}=N_{A} b \frac{T_{0}}{T} \rho_{x} \sum_{i}^{j} \frac{\sigma_{\text {nabs }, i}}{A_{i}} w_{i}
$$

where $N_{A}=6.022 \times 10^{23}$ is the Avogadro constant, the conversion factor $b=1 \times 10^{-24} \mathrm{~cm}^{2}, T_{0}=293 \mathrm{~K}$ represents the true thermal-neutron temperature, $T=120 \mathrm{~K}$ is the assumed near-thermal temperature of the BRR neutron beam. Clearly, for samples of mono-elemental composition $w_{i}=1$, in Eq. 6 above, and $\rho_{x}$ is simply replaced by $\rho_{i}$. The uncertainty in $\mu_{n}$, written as $\Delta_{\mu_{n}}$, is determined by propagating through the uncertainty on $\sigma_{\text {nabs }}$ alone, with all other terms in Eq. 6 treated as constants. This expression is strictly valid for a given neutronbeam temperature rather than a distribution. In practice, the neutrons will gain energy as they traverse the bulk volume of the sample, and for certain materials, a Monte Carlo calculation may be required to account for neutron thermalization over a disparate temperature range. However, for the tungsten oxide powders measured in this work, lowering the beam temperature by as much as $50 \mathrm{~K}$ only induces a change of the order of $\sim 1 \%$ in the overall attenuation.

Assuming $\mu_{\gamma}$ and $\mu_{n}$ to be independent variables, the overall uncertainty on the attenuation factor $I / I_{0}$ is then derived using the law of combination of errors

$$
\Delta_{I / I_{0}}^{2}=\left[\frac{\partial}{\partial \mu_{\gamma}}\left(\frac{I}{I_{0}}\right)\right]^{2} \Delta_{\mu_{\gamma}}^{2}+\left[\frac{\partial}{\partial \mu_{n}}\left(\frac{I}{I_{0}}\right)\right]^{2} \Delta_{\mu_{n}}^{2} .
$$

Appropriate error propagation and differentiating $I / I_{0}$ (Eq. 3) with respect to $\mu_{\gamma}$ and $\mu_{n}$, independently, yields the following expression quantifying the variance in $I / I_{0}$ from which the overall uncertainty may be deduced

$$
\begin{aligned}
\Delta_{I / I_{0}}^{2}= & {\left[\frac{1}{\frac{\mu_{\gamma}}{\cos \theta}+\frac{\mu_{n}}{\sin \theta}}\left(t e^{-\left(\frac{\mu_{\gamma}}{\cos \theta}+\frac{\mu_{n}}{\sin \theta}\right) t}-\frac{I}{I_{0}}\right)\right]^{2} } \\
& \times\left(\frac{\Delta_{\mu_{\gamma}}^{2}}{\cos ^{2} \theta}+\frac{\Delta_{\mu_{n}}^{2}}{\sin ^{2} \theta}\right) .
\end{aligned}
$$

\subsection{Standardization and determination of the effective sample thickness}

To establish the effective target thickness of the thick highdensity tungsten sample measured in this work, a set of absolute neutron-capture partial $\gamma$-ray production cross sections is required for comparison. Adopting comparator $\gamma$-ray transitions, whose cross sections are well known, permits use of an internalstandardization procedure, outlined in detail in Ref. [23]. In this procedure, the observed $\gamma$-ray intensities may be normalized by scaling to the well-known comparator transitions observed in the same capture- $\gamma$ spectrum. For comparative purposes here, we used strong $\gamma$ lines in ${ }^{187} \mathrm{~W}$ that were extracted from a previous thin-sample measurement of a low-density hydrated tungstic acid $\left(\mathrm{H}_{2} \mathrm{WO}_{4}\right)$ compound of natural composition [8]. In that measurement, the absolute cross sections for the ${ }^{187} \mathrm{~W} \gamma$ lines, listed in Table 1, were extracted via internal standardization to the $2223-\mathrm{keV}$ transition in hydrogen 

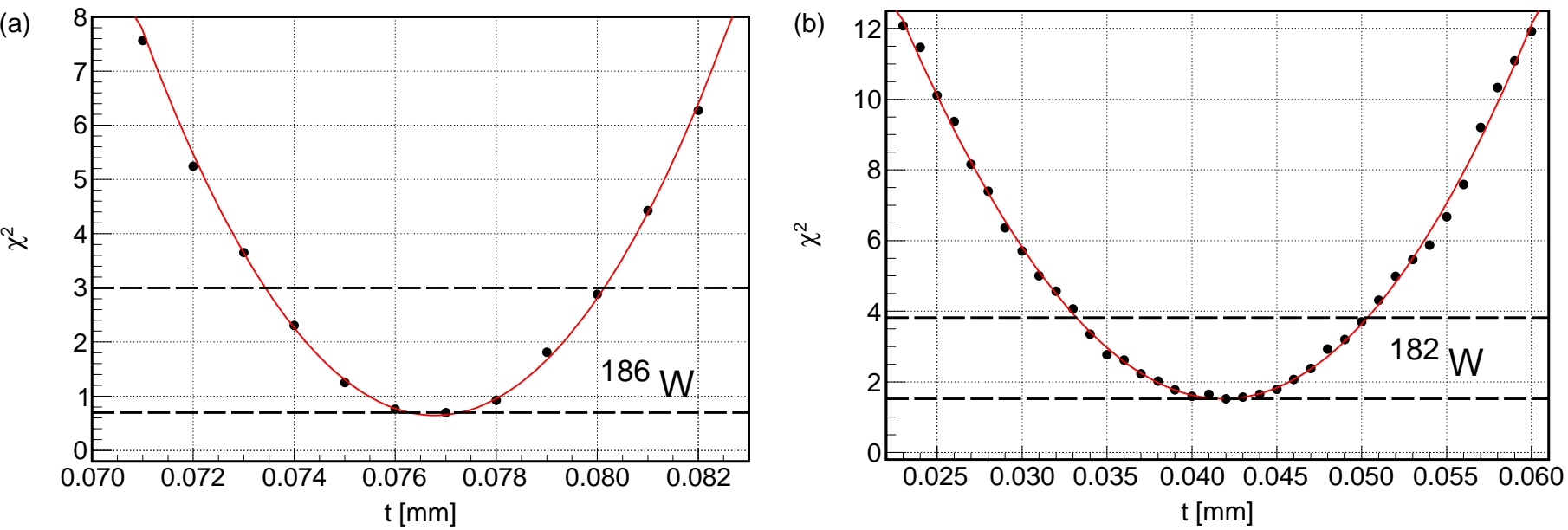

Figure 2: Plots showing the global $\chi^{2}$ distribution as a function of thickness $(t)$ for the ${ }^{186} \mathrm{~W}$ (a) and ${ }^{182} \mathrm{~W}$ (b) samples measured in this work. The lower-dashed line in each plot indicates the minima of the respective distributions and corresponds to the mean effective target thickness: (a) $\chi_{\min }^{2} \approx 0.70(\mathrm{ndf}=2)$ at $t=0.077 \mathrm{~mm}$; (b) $\chi_{\min }^{2} \approx 1.5(\mathrm{ndf}=3)$ at $t=0.042 \mathrm{~mm}$. The upper-dashed line is drawn at $\chi_{\min }^{2}+2.3 \mathrm{in}$ each plot to illustrate the $1 \sigma$ acceptable range of $t$ values consistent with the uncertainty window $(\Delta t)$ of the deduced effective target thickness.

which has a well-known partial $\gamma$-ray production cross section $\sigma_{\gamma}(2223 \mathrm{keV})=0.3326(7) \mathrm{b}$ [23], and taking into account the stoichiometric ratio of $\mathrm{H}: \mathrm{W}$. The lack of any strong $s$ wave resonances near the thermal-neutron point at $25.3 \mathrm{meV}$ in tungsten renders applicable a pure $1 / v$ dependence on the observed cross section near and below thermal-neutron energies $\left(E_{n}\right)$. Under these circumstances, the cross section may be represented as shown in Ref. [24], accordingly:

$$
\sigma_{\gamma}\left(E_{n} ; v\right)=\sigma_{0} \sqrt{\left(\frac{E_{0}}{E_{n}}\right)}=\sigma_{0}\left(\frac{v_{0}}{v}\right),
$$

where $\sigma_{0}$ is the total radiative thermal neutron-capture cross section, $v_{0}=2200 \mathrm{~m} / \mathrm{s}$ is the thermal neutron velocity, and $E_{0}=25.3 \mathrm{meV}$. It follows, therefore, that

$$
\sigma_{\gamma}\left(E_{n}\right) \propto \frac{1}{v} \quad \forall \quad E_{n} \lesssim 25.3 \mathrm{meV} .
$$

Also, since $\left(T_{0} / T\right)=\sqrt{\left(E_{0} / E\right)}$, at our assumed average beam temperature $T=120 \mathrm{~K}$, the $1 / v$ law implies a corresponding neutron-beam energy centered around $4.2 \mathrm{meV}$. Thus, although Eqs. 9 and 10 are rigorously defined for monoenergetic neutrons [24], our measurement concerns a distribution of neutrons centered on $\sim 4.2 \mathrm{meV}$. However, because both tungsten and hydrogen obey the $1 / v$ law (i.e. Eqs. 9 and 10 are valid), moreover $\sigma_{\gamma}(\mathrm{W})$ are deduced relative to $\sigma_{\gamma}(\mathrm{H})$, it follows that any correction for the neutron-beam energy cancels.

The absolute cross sections, $\sigma_{\gamma}^{\mathrm{S}}$, of Table 1 [8] were adopted for standardization purposes since they cover the complete energy range of interest: from below $100 \mathrm{keV}$, where $\gamma$-ray self absorption is significant, to several $\mathrm{MeV}$, where absorption is negligible. In general, the measured peak areas $A_{\gamma}$ observed in the capture- $\gamma$ spectrum are related to their corresponding transition cross sections according to the expression

$$
A_{\gamma}=\sigma_{\gamma} \frac{m N_{A}}{M} \theta \epsilon_{\gamma}\left(E_{\gamma}\right) \frac{I\left(E_{\gamma}\right)}{I_{0}} \phi \tau g_{\mathrm{W}}(T),
$$

where $m$ (the sample mass), $N_{A}$, and $M$ are described in Sect. 2.1, $\theta$ is the isotopic abundance, $\phi$ is the neutron flux, $\tau$ is the data-acquisition deadtime-corrected irradiation period, and $\epsilon_{\gamma}\left(E_{\gamma}\right)$ denotes the relative $\gamma$-ray energy-dependent detection efficiency. The detection efficiency represents a total efficiency given by the product of the intrinsic-detection efficiency and the geometric efficiency describing the solid angle subtended by the detector with respect to the sample position. The temperaturedependent Westcott $g_{\mathrm{W}}$ factor, $g_{\mathrm{W}}(T)$, is a correction factor used to describe non- $1 / v$ behaviour exhibited by irregular nuclides [24]; $g_{\mathrm{W}}$ is given by the ratio of the effective cross section for a pure Maxwellian spectrum at a given $T$ to the $2200-\mathrm{m} / \mathrm{s}$ (i.e. $T=293 \mathrm{~K}$ ) cross section [25]. The $g_{\mathrm{W}}$ factors for the hydrogen and tungsten isotopes considered in this study do not deviate from unity by more than $1 \%$ at $T=120 \mathrm{~K}$ or at $T=293 \mathrm{~K}$ [25], i.e. $g_{\mathrm{W}} \approx 1$ and no correction to $A_{\gamma}$ is needed. Because $m$, $N_{A}, M, \theta, \phi, \tau$, and $T$ are systematic quantities that contribute to the observed count rate in all $\gamma$ lines, collectively they constitute a constant $K$, such that

$$
\frac{A_{\gamma}^{\prime}}{\sigma_{\gamma}} \cdot \frac{1}{I\left(E_{\gamma}\right) / I_{0}}=K
$$

where $A_{\gamma}^{\prime}=A_{\gamma} / \epsilon_{\gamma}\left(E_{\gamma}\right)$ is the efficiency-corrected peak area. Although both $\epsilon_{\gamma}$ and the attenuation factor $I / I_{0}$ are dependent on $E_{\gamma}$, since the measured peak area for a given transition is directly proportional to its corresponding absolute standard cross section, it follows that the ratio of $\sigma_{\gamma}^{\mathrm{S}}$ to the measured peak area will be constant for any transition provided the peak areas have been appropriately corrected for these quantities. This condition may be expressed as

$$
\frac{\sigma_{\gamma_{1}}^{\mathrm{S}}\left(E_{\gamma_{1}}\right)}{A_{\gamma_{1}}^{c}\left(E_{\gamma_{1}}\right)}=\frac{\sigma_{\gamma_{2}}^{\mathrm{S}}\left(E_{\gamma_{2}}\right)}{A_{\gamma_{2}}^{c}\left(E_{\gamma_{2}}\right)}=\frac{\sigma_{\gamma_{3}}^{\mathrm{S}}\left(E_{\gamma_{3}}\right)}{A_{\gamma_{3}}^{c}\left(E_{\gamma_{3}}\right)}=\cdots,
$$

or, in general terms,

$$
R_{i}\left(E_{\gamma_{i}}\right)=\frac{\sigma_{\gamma_{i}}^{\mathrm{S}}\left(E_{\gamma_{i}}\right)}{A_{\gamma_{i}}^{c}}=\text { const. }
$$


where $A_{\gamma}^{c}$ represents the attenuation-corrected measured peak area given by

$$
A_{\gamma}^{c}=\frac{A_{\gamma}^{\prime}}{I / I_{0}(t)}
$$

and $A_{\gamma}^{\prime}$ is the $\epsilon_{\gamma}$-corrected attenuated peak area measured directly in the capture- $\gamma$ spectrum. The effective sample thickness is then determined by treating $t$ as an adjustable parameter in Eq. 3 and varying this quantity until the condition embodied by the ratio in Eqs. 13 and 14 converges upon a unique result. An appropriate energy-dependent attenuation correction can then be applied to the entire capture- $\gamma$ spectrum. Uncertainties for all relevant quantities are determined and propagated in the standard manner; they are also listed in Table 1.

For a given sample, since the ratios $R_{i}\left(E_{\gamma_{i}}\right)$ are not independent measurements but are in fact correlated, a covariance matrix $\mathbf{V}$, containing the associated uncertainties $\Delta_{R_{i}}$, is needed to describe them. From the principle of maximum likelihood we know that the probability of a particular set of data is the product of the individual probabilities, and this provides a basis for the least squares method adopted in this analysis. By minimizing the exponent in the Gaussian likelihood function we may determine the global $\chi^{2}$ for an assumed effective thickness $t$ using the following expression

$$
\chi^{2}=\sum_{i=1}^{N} \sum_{j=1}^{N}\left[R_{w}-R_{i}\left(E_{\gamma_{i}}\right)\right]\left[V_{i j}^{-1}\right]\left[R_{w}-R_{j}\left(E_{\gamma_{j}}\right)\right],
$$

where $R_{w}$ is the expectation value at a given $t$ determined from the weighted average of the corresponding set of $R_{i}\left(E_{\gamma_{i}}\right)$ measurements. The individual $(i, j)$ elements of an $N \times N$ covariance matrix, where $N$ represents the maximum number of data points, are given as $V_{i j}=\Delta_{R_{i}}^{2} \delta_{i j}$ for the diagonal elements (where the Kronecker delta function $\delta_{i j}=1$ for $i=j$, and 0 for $i \neq j$ ) and $V_{i j}=r_{i j} \Delta_{R_{i}} \Delta_{R_{j}}$ for the off-diagonal matrix elements, where $r_{i j}$ is the correlation coefficient between a pair of $R_{i}\left(E_{\gamma_{i}}\right)$ measurements. For each of the $N$ data points the corresponding ratios $R_{1}\left(E_{\gamma_{1}}\right), R_{2}\left(E_{\gamma_{2}}\right), \ldots, R_{N}\left(E_{\gamma_{N}}\right)$, may be denoted $\mathbf{R}$, where $\mathbf{R}$ is a vector of $N$ elements. Writing $\mathbf{R}_{\mathbf{w}}$ also as an $N$-element vector then allows Eq. 16 to be conveniently recast in matrix notation as

$$
\chi^{2}=\left(\mathbf{R}_{\mathbf{w}}-\mathbf{R}\right) \mathbf{V}^{-1}\left(\widetilde{\mathbf{R}_{w}}-\tilde{\mathbf{R}}\right)
$$

where $\widetilde{\mathbf{R}_{\mathbf{w}}}$ and $\tilde{\mathbf{R}}$ represent the transposed vectors $\mathbf{R}_{\mathbf{w}}$ and $\mathbf{R}$, respectively.

In this work, two parameters $(P)$ are adjusted to minimize $\chi^{2}$ to fit the $N$ data points: $t$ and $r_{i j}$. Here, $r_{i j}$ represents an average solution. The number of degrees of freedom is given by ndf $=N-P$. For the ${ }^{186} \mathrm{~W}$ and ${ }^{182} \mathrm{~W}$ measurements $N=4$ and 5 , respectively, thus ndf $=2$ and 3 , respectively. According to Poisson statistics the reduced $\chi^{2}$, i.e. $\chi^{2} / \mathrm{ndf}$, for distributions with ndf $=2$ and 3 are expected to be $\sim 0.35$ and $\sim 0.5$, respectively, at the $68.3 \%$ confidence level [26]. The corresponding value of $t$ yielding a global $\chi_{\min }^{2}$, at values of approximately 0.7 (ndf $=2$ ) and 1.5 (ndf = 3), for the fit from a two-parameter adjustment may then be interpreted as the mean effective sample thickness. Its uncertainty $\Delta t$ can then be extracted using

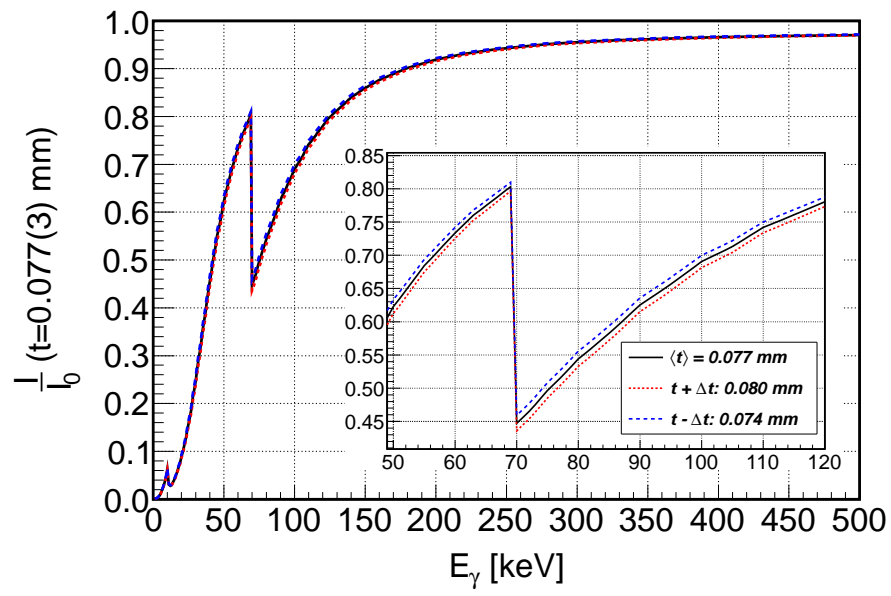

Figure 3: The $\gamma$-ray energy-dependent attenuation factor, $I / I_{0}$, plotted as a function of $E_{\gamma}$ for the ${ }^{186} \mathrm{~W}$ sample assuming $t=0.077$ (3) $\mathrm{mm}$ in Eq. 3. The curve is expanded around $E_{\gamma}=50-120 \mathrm{keV}$ to reveal the upper and lower bounds of the uncertainty band (for one standard deviation). This curve provides an $E_{\gamma}$-dependent correction to the measured transition intensities in the capture- $\gamma$ spectrum. The abrupt $K$-edge in tungsten is clearly visible at around $69-70 \mathrm{keV}$ and corresponds to a sharp increase in $\mu_{\gamma}$ at the $K$-shell electron binding energy; the smaller cumulative effect of the $L$-I, $L$-II, and $L$-III edges is also observed in the low-energy regime spanning approximately $10-12 \mathrm{keV}$.

the method of least squares from the range of values for $t \pm \Delta t$ satisying the condition $\chi^{2} \leq \chi_{\min }^{2}+2.3$ [26]. Accordingly, we estimate $\Delta t$ using

$$
\Delta t=\frac{1}{2}\left[t_{>}\left(\chi_{\min }^{2}+2.3\right)-t_{<}\left(\chi_{\min }^{2}+2.3\right)\right]
$$

where $t_{>}$and $t_{<}$are the interpolated values of $t$ at $\chi_{\min }^{2}+2.3$, with $t_{>}>t$ and $t_{<}<t$.

\section{Results}

To illustrate the procedure for determination of the effective thickness $t$, we present results from an $(n, \gamma)$ measurement for a 169 -mg powder sample of ${ }^{186} \mathrm{WO}_{2}(99.65(3) \%$ enrichment) irradiated for a period of $2.03 \mathrm{~h}$ to induce prompt radioactivity in the ${ }^{187} \mathrm{~W}$ compound nucleus [8]. Alongside the standardizationtransition energies and cross sections listed in Table 1 are the corresponding $\epsilon_{\gamma}$-corrected attenuated peak areas, $A_{\gamma}^{\prime}$. Using this data, we iteratively step through values of $t$ over a suitable range of thicknesses to calculate $A_{\gamma_{i}}^{c}$ (Eq. 15) to find a statistically consistent $R_{i}\left(E_{\gamma_{i}}\right)$ (Eq. 14$)$ data set. Simultaneously, we adjust $r_{i j}$ between 0 and 1 to minimize the $\chi^{2}$ function in Eq. 17. Figure 2(a) shows the global $\chi^{2}$ distribution plotted as a function of $t$ and reveals a tungsten effective sample thickness $t=0.077 \mathrm{~mm}$ (equivalent to $0.16 \mathrm{~mm}$ for $\mathrm{WO}_{2}$ ), assuming an overall average correlation coefficient $r_{i j}=0.82$, can reproduce $\chi_{\min }^{2}=0.70$ i.e. the global $\chi^{2}$ expectation value for a minimzation based on ndf $=2$. The corresponding results for $A_{\gamma_{i}}^{c}$ and $R_{i}\left(E_{\gamma_{i}}\right)$ at $t=0.077 \mathrm{~mm}$ are also summarized in Table 1 , from which an average $R_{w}=9.04 \times 10^{-6} \mathrm{~b} \cdot \mathrm{cnt}^{-1}$ is determined. The elements of the covariance matrix supporting this result may be written as a product of the correlation coefficient and the associated $R_{i}\left(E_{\gamma_{i}}\right)$ uncertainty products, $\Delta_{R_{i}}$ from Table 1 . Because 
Table 2: Standard cross sections, $\sigma_{\gamma}^{\mathrm{S}}$, in ${ }^{186} \mathrm{~W}(n, \gamma){ }^{187} \mathrm{~W}$ and ${ }^{182} \mathrm{~W}(n, \gamma){ }^{183} \mathrm{~W}$ are derived from the $\mathrm{H}_{2} \mathrm{WO}_{4}$ normalization measurement $[8]$ and the attenuated cross sections, $\sigma_{\gamma}^{\mathrm{T}}$, are from the thick-sample measurements using enriched ${ }^{186} \mathrm{~W}$ and ${ }^{182} \mathrm{~W}$, respectively. Cross sections are corrected for isotopic abundances: ${ }^{186} \mathrm{~W}$ $(28.43 \%)$ and ${ }^{182} \mathrm{~W}(26.50 \%)$ [27]. The experimental attenuation factors $\left(I / I_{0}\right)_{\text {exp }}$ were deduced from the ratio $\sigma_{\gamma}^{\mathrm{T}} / \sigma_{\gamma}^{\mathrm{S}}($ Eq. 19$)$ and compared to the theoretical values $I / I_{0}$ (Eq. 3) using effective thicknesses of $0.077 \mathrm{~mm}\left({ }^{186} \mathrm{~W}\right)$ and $0.042 \mathrm{~mm}\left({ }^{182} \mathrm{~W}\right)$, assuming: (1) both $\gamma$-ray absorption and neutron self shielding; (2) $\gamma$-ray absorption only. Uncertainties are smaller in the first case owing to the overall non-linearity represented by the combination $\mu_{\gamma}$ and $\mu_{n}$ according to the functional orm of Eq. 8.

\begin{tabular}{|c|c|c|c|c|c|c|}
\hline \hline Sample & $E_{\gamma}[\mathrm{keV}]$ & $\sigma_{\gamma}^{\mathrm{S}}[\mathrm{b}]$ & $\sigma_{\gamma}^{\mathrm{T}}[\mathrm{b}]$ & $\left(\frac{I}{I_{0}}\right)_{\exp }$ & $\left(\frac{I}{I_{0}}\right)_{\left\langle\mu_{\gamma}+\mu_{n}\right\rangle}{ }^{1}$ & $\left(\frac{I}{I_{0}}\right)_{\left\langle\mu_{\gamma}\right\rangle}{ }^{2}$ \\
\hline${ }^{186} \mathrm{~W}$ & $77.39(3)$ & $0.823(14)$ & $0.433(14)$ & $0.526(19)$ & $0.514(25)$ & $0.522(26)$ \\
& $145.79(3)$ & $4.727(46)$ & $4.05(20)$ & $0.856(44)$ & $0.849(37)$ & $0.867(43)$ \\
& $273.10(5)$ & $1.337(14)$ & $1.26(13)$ & $0.942(96)$ & $0.943(30)$ & $0.963(48)$ \\
& $5261.68(6)$ & $2.297(32)$ & $2.297(64)$ & $1.000(31)$ & $0.975(15)$ & $0.997(49)$ \\
\hline \hline \multirow{2}{*}{$182 \mathrm{~W}$} & $98.90(1)$ & $0.342(12)$ & $0.282(7)$ & $0.825(36)$ & $0.806(38)$ & $0.815(41)$ \\
& $162.11(1)$ & $0.983(27)$ & $0.912(21)$ & $0.928(33)$ & $0.928(39)$ & $0.939(47)$ \\
& $291.57(1)$ & $0.251(9)$ & $0.252(6)$ & $1.003(44)$ & $0.973(28)$ & $0.985(49)$ \\
& $1100.39(3)$ & $0.126(5)$ & $0.128(4)$ & $1.019(54)$ & $0.985(16)$ & $0.997(50)$ \\
& $6190.78(6)$ & $2.740(38)$ & $2.740(66)$ & $1.000(28)$ & $0.986(15)$ & $0.998(50)$ \\
\hline \hline
\end{tabular}

$N=4$, the general form of the $4 \times 4$ covariance matrix may be represented as

$$
V=\left(\begin{array}{llll}
r_{11} \Delta_{R_{1}} \Delta_{R_{1}} & r_{12} \Delta_{R_{1}} \Delta_{R_{2}} & r_{13} \Delta_{R_{1}} \Delta_{R_{3}} & r_{14} \Delta_{R_{1}} \Delta_{R_{4}} \\
r_{21} \Delta_{R_{2}} \Delta_{R_{1}} & r_{22} \Delta_{R_{2}} \Delta_{R_{2}} & r_{23} \Delta_{R_{2}} \Delta_{R_{3}} & r_{24} \Delta_{R_{2}} \Delta_{R_{4}} \\
r_{31} \Delta_{R_{3}} \Delta_{R_{1}} & r_{32} \Delta_{R_{3}} \Delta_{R_{2}} & r_{33} \Delta_{R_{3}} \Delta_{R_{3}} & r_{34} \Delta_{R_{3}} \Delta_{R_{4}} \\
r_{41} \Delta_{R_{4}} \Delta_{R_{1}} & r_{42} \Delta_{R_{4}} \Delta_{R_{2}} & r_{43} \Delta_{R_{4}} \Delta_{R_{3}} & r_{44} \Delta_{R_{4}} \Delta_{R_{4}}
\end{array}\right),
$$

where, for the diagonal matrix elements $r_{i j}=1$ (see Sect. 2.2), and for all off-diagonal elements $r_{i j}=0.82$. Thus, using these values for $r_{i j}$ together with the corresponding indexed uncertainties from the final column of Table 1 yields

$$
V=\left(\begin{array}{lll}
{[1.00 \times 0.27 \times 0.27]} & \cdots & {[0.82 \times 0.27 \times 0.20]} \\
{[0.82 \times 0.42 \times 0.27]} & \cdots & {[0.82 \times 0.42 \times 0.20]} \\
{[0.82 \times 0.92 \times 0.27]} & \cdots & {[0.82 \times 0.92 \times 0.20]} \\
{[0.82 \times 0.20 \times 0.27]} & \cdots & {[1.00 \times 0.20 \times 0.20]}
\end{array}\right) \text {. }
$$

For clarity, we have factored through the $10^{-6}$ common denominator associated with all $R_{i}\left(E_{\gamma_{i}}\right)$ and $\Delta_{R_{i}}$ values of Table 1 , thus simplifying the $\chi^{2}$ calculation.

The overall uncertainty on the target thickness is extracted from the global $\chi^{2}$ distribution and interpolating the range of $t$ consistent with $\chi^{2} \leq \chi_{\min }^{2}+2.3 \lesssim 3$. Figure 2 (a) shows that values of $t$ between approximately 0.0734 and $0.0801 \mathrm{~mm}(3 \mathrm{sf})$ satisfy this criterion window of $\chi^{2} \lesssim 3$. Hence, from Eq. 18 we report an estimate for $\Delta t= \pm 0.003 \mathrm{~mm}(3 \mathrm{dp})$. Our result for the effective target thickness of the inhomogeneous powder sample, therefore, describes the equivalent planar thickness of the tungsten sample based on the measured apparent overall self attenuation within the sample. The one-standard-deviation $\gamma$-ray energy-dependent attenuation correction, applicable to the observed $\gamma$-ray intensities measured in the $(n, \gamma)$ capture spectrum [8], corresponding to an effective thickness $t=0.077$ (3) $\mathrm{mm}$ is shown in Fig. 3. From a similar analysis carried out on a 274-mg ${ }^{182} \mathrm{WO}_{2}$ sample (92.7(9)\% enrichment) irradiated for $2.46 \mathrm{~h}$, we established the global $\chi_{\min }^{2}=1.5$ (the expectation value based on ndf $=3$ ) using $r_{i j}=0.60$ to extract an effective sample thickness $t=0.042(8) \mathrm{mm}$ for ${ }^{182} \mathrm{~W}$ (equivalent to $0.087 \mathrm{~mm}$ for $\mathrm{WO}_{2}$ ) as shown in Fig. 2(b).

As a consistency check, the overall attenuation factors may also be deduced experimentally by comparing the attenuated partial $\gamma$-ray production cross sections from the thick highdensity ${ }^{186} \mathrm{~W}$ and ${ }^{182} \mathrm{~W}$ measurements, referred to as $\sigma_{\gamma}^{\mathrm{T}}$, to the same transitions from the reference standard with known absolute cross sections, $\sigma_{\gamma}^{\mathrm{S}}$. A similar procedure is described in Ref. [4]. The attenuated $\sigma_{\gamma}^{\mathrm{T}}$ values are also deduced via the internal-standardization procedure (see Sect. 2.2 and references therein): for the ${ }^{186} \mathrm{~W}$ sample we scaled to the high-energy comparator line in ${ }^{187} \mathrm{~W}$ at $5261.68 \mathrm{keV}$; for the ${ }^{182} \mathrm{~W}$ sample we used the $6190.78-\mathrm{keV}$ line in ${ }^{183} \mathrm{~W}$. Both high-energy primary $\gamma$ rays are subject to negligible absorption. Because the thicksample cross sections, $\sigma_{\gamma}^{\mathrm{T}}$, have not been corrected for self attenuation, and $\sigma_{\gamma}^{\mathrm{S}}$ represents the ideal standard for a given transition, the experimental attenuation factor, $\left(I / I_{0}\right)_{\text {exp }}$, may be defined by the following ratio

$$
\left(\frac{I}{I_{0}}\right)_{\exp }=\frac{\sigma_{\gamma}^{\mathrm{T}}}{\sigma_{\gamma}^{\mathrm{S}}} .
$$

Clearly, for lower-energy transitions the observed attenuation 

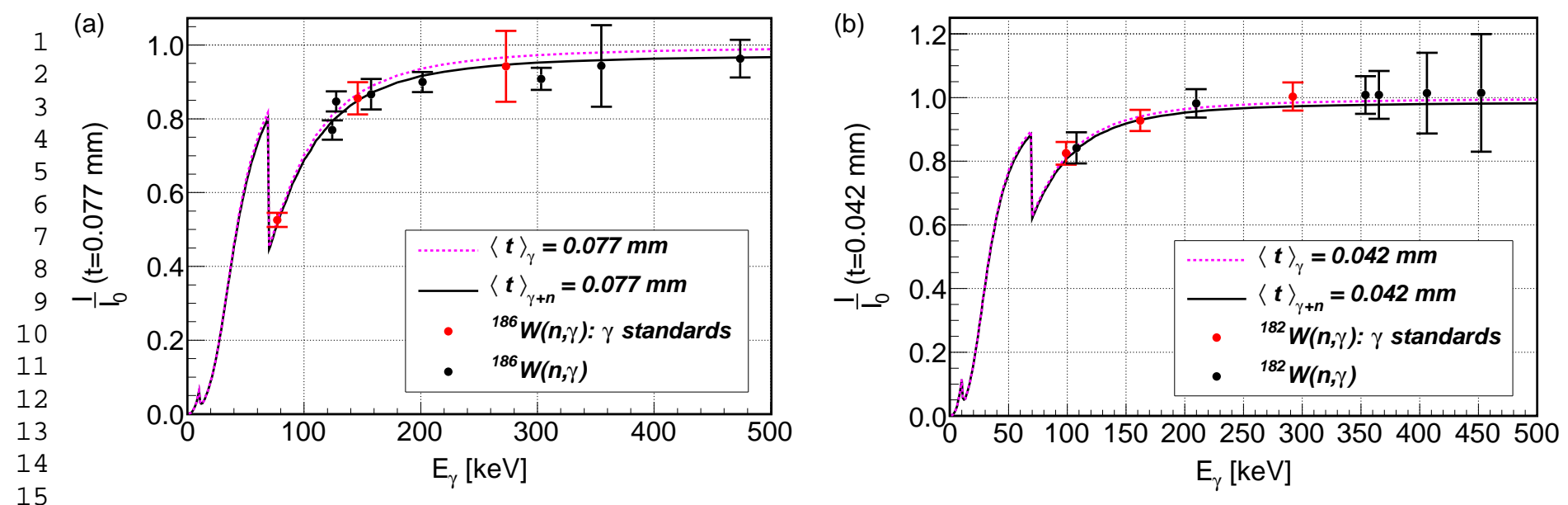

Figure 4: The $\gamma$-ray energy-dependent attenuation factor, $I / I_{0}$, plotted as a function of $E_{\gamma}$ for energies up to $500 \mathrm{keV}$, assuming: (a) $t=0.077 \mathrm{~mm}\left({ }^{186} \mathrm{~W}\right.$ ); (b) $t=0.042 \mathrm{~mm}\left({ }^{182} \mathrm{~W}\right)$. The solid-black curve corresponds to $I / I_{0}$ as a function of both $\mu_{\gamma}$ and $\mu_{n}$, whereas the dashed-magenta curve neglects the $\mu_{n}$ dependence i.e. $I / I_{0}$ as a function of $\mu_{\gamma}$ alone. The data points show the experimentally-deduced attenuation factors for individual $\gamma$-ray transitions using Eq. 19: the red points correspond to transitions used to deduce the ${ }^{186} \mathrm{~W}$ and ${ }^{182} \mathrm{~W}$ sample thicknesses; the black data points were not used in the derivation of the sample thicknesses but only for comparison.

is more apparent and although $\sigma_{\gamma}^{\mathrm{T}}<\sigma_{\gamma}^{\mathrm{S}}$ for all transitions, $\sigma_{\gamma}^{\mathrm{T}}$ asymptotically reaches $\sigma_{\gamma}^{\mathrm{S}}$ as $E_{\gamma}$ increases beyond $\gtrsim 300 \mathrm{keV}$ (where $I / I_{0} \rightarrow 1$ ). Presented in Table 2 are the standardized thick-sample cross sections, corrected for the respective isotopic abundances of ${ }^{186} \mathrm{~W}(28.43 \%)$ and ${ }^{182} \mathrm{~W}(26.50 \%)$ [27], and the corresponding experimental attenuation factors. The effective sample thicknesses extracted from the covariance analyses may then be compared to the observed experimental values given by Eq. 19 for each $E_{\gamma}$. As shown in Table 2, the theoretical values for $I / I_{0}$, assuming $t=0.077 \mathrm{~mm}$ for the ${ }^{186} \mathrm{~W}$ sample and $t=0.042 \mathrm{~mm}$ for ${ }^{182} \mathrm{~W}$ sample, compare favourably with the experimentally-deduced values for each $E_{\gamma}$. Remarkable consistency is achieved assuming: (i) both $\gamma$-ray self absorption and neutron self shielding (Eq. 3); (ii) $\gamma$-ray self absorption alone (Eq. 3 without the $\mu_{n}$ dependence). In the thick-sample approximation, the irradiated sample absorbs all incident neutrons allowing the $\gamma$-ray absorption to be treated independently. Our results in this case reveal very little difference in the calculation of $I / I_{0}\left(\mu_{\gamma}, \mu_{n}\right)$ and $I / I_{0}\left(\mu_{\gamma}\right)$ at the assumed sample thicknesses and both sets of calculations are in excellent agreement with the experimentally-deduced attenuations. This agreement is reinforced in Fig. 4 which shows excellent consistency between calculated and experimental attenuation factors, not only for the transitions used in deriving the effective sample thickness (red data points), but also for other prominent transitions (black data points) that may be used for comparison between the standard $\left(\mathrm{H}_{2} \mathrm{WO}_{4}\right)$ and enriched thick-target $(n, \gamma)$ spectra. This observation provides a useful verification of the adopted method for the determination of the effective sample thickness.

\section{Conclusion}

A capability has been demonstrated to precisely determine effective sample thicknesses for irregular geometries with nonuniform areal densities through radiative capture. This technique is predicated on quantifying the $\gamma$-ray absorption ob- served in low-energy transitions by comparison to known absolute partial $\gamma$-ray production cross sections, and has been experimentally verified through neutron-beam $(n, \gamma)$ measurements performed with the PGAA setup at the BRR. We have successfully illustrated this concept to a precision of better than $4 \%$ for a 0.077 (3)-mm measurement of the tungsten-equivalent effective sample thickness for an enriched ${ }^{186} \mathrm{WO}_{2}$ powdered sample, and we have also measured a tungsten-equivalent effective sample thickness of $0.042(8) \mathrm{mm}$ for an enriched ${ }^{182} \mathrm{WO}_{2}$ powdered sample. The level of precision attained may be explained by the sensitivity of the $\gamma$-ray yield for low-energy transitions with respect to the assumed sample thickness. In the case of ${ }^{186} \mathrm{~W}$, the strong well-resolved low-energy transition at $\sim 77 \mathrm{keV}$ provides enhanced sensitivity for this adopted methodology.

For materials, such as tungsten, with low-to-moderate neutron-capture cross sections, neutrons are more likely to penetrate deeper into the sample, and so capture-reaction radioactivity is likely to be induced from deeper within the bulk-sample volume. Consequently, the transmission path through which the $\gamma$ rays must traverse, on average, is likely to represent the mean thickness of the irradiated sample. Because tungsten is also a high- $\rho$ density material, the probability of complete photoelectric absorption within the sample is also high leading to large corrections, particularly for the lower-energy ( $\lesssim 300 \mathrm{keV})$ transitions that are subject to higher degrees of $\gamma$-ray self absorption. The amount of overall attenuation, therefore, provides a measure of the apparent, effective, thickness of the sample volume illuminated by the neutron beam. In general, this measured effective thickness is likely to be somewhat less than the true thickness represented by the entirety of the bulk sample material, and the amount of absorption and self shielding from an integration over the true thickness may constitute an over estimate of the correction factor. Furthermore, $\gamma$-ray transmission through samples with high neutron-capture cross sections, or low- $\rho$ materials, is far less likely to be impeded to a measur- 
able extent. However, future measurements are needed to help understand the limitations, and useful implementations, of this technique.

\section{Acknowledgements}

This work was performed under the auspices of the University of California, supported by the Director, Office of Science, Office of Basic Energy Sciences, of the U. S. Department of Energy at the Lawrence Berkeley National Laboratory under Contract DE-AC02-05CH11231, and by the U. S. Department of Energy by the Lawrence Livermore National Laboratory under Contract DE-AC52-07NA27344. The access to the Budapest PGAA facility was financially supported by the NAP VENEUS08 grant under Contract OMFB-00184/2006. The operations staff at the Budapest Research Reactor are gratefully acknowledged. One of the authors (AH) would like to thank Dr. W. Younes for insightful physics discussions.

\section{References}

[1] J. T. Goorley et al., Initial MCNP6 Release Overview - MCNP6 version 1.0, LA-UR-13-22934 (2013); https : //mcnp. lanl.gov/.

[2] S. Agostinelli et al., Nucl. Instrum. and Methods Phys. Res. Sect. A 506 (2003) 250.

[3] M. Blaauw and T. Belgya, J. Radioanal. Nucl. Chem. 265 (2005) 257.

[4] A. Borella et al., J. Radioanal. Nucl. Chem. 265 (2005) 267.

[5] Z. Kis, T. Belgya, and L. Szentmiklósi, Nucl. Instrum. and Methods Phys. Res. Sect. A 638 (2011) 143.

[6] Zs. Révay et al., Nucl. Instrum. and Methods Phys. Res. Sect. B 213 (2004) 385.

[7] L. Szentmiklósi et al., J. Radioanal Nucl. Chem. 286 (2010) 501.

[8] A. M. Hurst et al., Phys. Rev. C 89 (2014) 014606.

[9] R. B. Firestone, Database of Prompt Gamma Rays from Slow Neutron Capture for Elemental Analysis (International Atomic Energy Agency, Vienna, 2006); https://www-nds.iaea.org/pgaa/egaf.html

[10] Zs. Révay, R. B. Firestone, T. Belgya, and G. L. Molnár, Handbook of Prompt Gamma Activation Analysis (Kluwer Academic Publishers, The Netherlands, 2004), chap. Prompt Gamma-Ray Spectrum Catalog p. 173.

[11] T. Belgya et al. (Springer Verlag, Budapest, Hungary, 1997), Proc. 9th International Symposium on Capture Gamma-Ray Spectroscopy and Related Topics, p. 826.

[12] L. Rosta et al., Physica B 234-236 (1997) 1196.

[13] L. Rosta, Appl. Phys. A 74 (2002) S52-S54.

[14] L. Rosta, T. Grósz, and T. Hargitai, Appl. Phys. A 74 (2002) S240-S242.

[15] T. Belgya, Phys. Proc. 31 (2012) 99.

[16] T. Belgya, Z. Kis, and L. Szentmiklósi, Nucl. Data Sheets 119 (2014) 419.

[17] B. Fazekas et al., J. Radioanal. Nucl. Chem. 215 (1997) 271.

[18] Zs. Révay, Anal. Chem. 81 (2009) 6851.

[19] R. Nowotny, XMuDat: Photon attenuation data on PC (IAEA-NDS-195, Version 1.0.1, August 1998); https://www-nds.iaea.org/publications/iaea-nds/ iaea-nds-0195.htm

[20] J. H. Hubbel and S. M. Seltzer, Tables of X-Ray Mass Attenuation Coefficients and Mass Energy-Absorption Coefficients $1 \mathrm{keV}$ to $20 \mathrm{MeV}$ for Elements $Z=1$ to 92 and 48 Additional Substances of Dosimetric Interest (NISTIR 5632, 1995); http://www.nist.gov/pml/data/xraycoef/index.cfm/

[21] J. M. Boone and A. E. Chavez, Med. Phys. 23, 12 (1996) 1997.

[22] S. F. Mughabghab, Atlas of Neutron Resonances: Resonance Parameters and Thermal Cross Sections $Z=1-100$ (Elsevier BV, New York, 2006) 5 th ed.

[23] Zs. Révay and G. L. Molnár, Radiochim. Acta 91 (2003) 361.
[24] Zs. Révay and T. Belgya, Handbook of Prompt Gamma Activation Analysis (Kluwer Academic Publishers, The Netherlands, 2004), chap. Principles of the PGAA method, p. 1.

[25] H. D. Choi and T. Trkov, Database of Prompt Gamma Rays from Slow Neutron Capture for Elemental Analysis (International Atomic Energy Agency, Vienna, 2007), chap. Nomenclature, Westcott $g_{\mathrm{W}}$ Factors and Neutron Spectral Shape Dependent Formalism, p. 5.

[26] J. Beringer et al. (Particle Data Group), Phys. Rev. D 86 (2012) 010001; G. Cowan, Statistics, p. 390-401.

[27] M. Berglund and M. Wiesser, Pure Appl. Chem. 83 (2011) 397. 
Figure2 $\vdots$
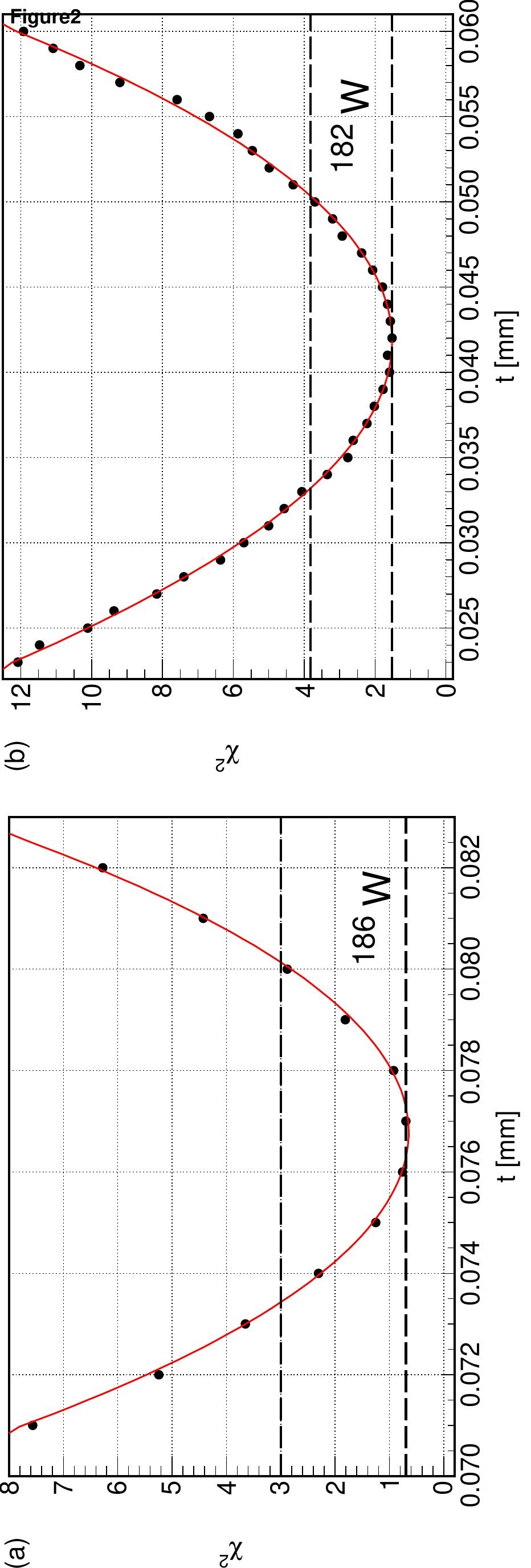


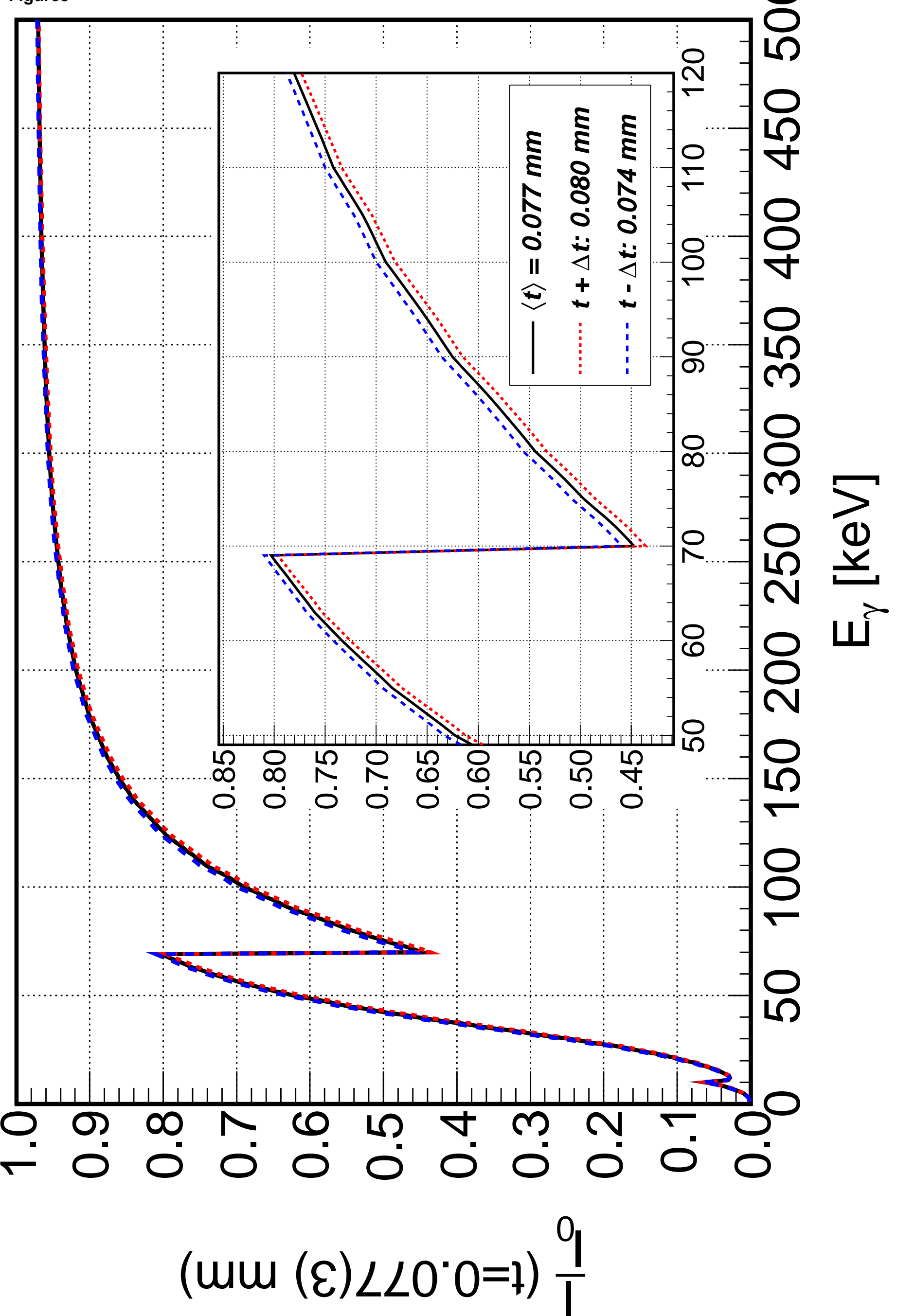




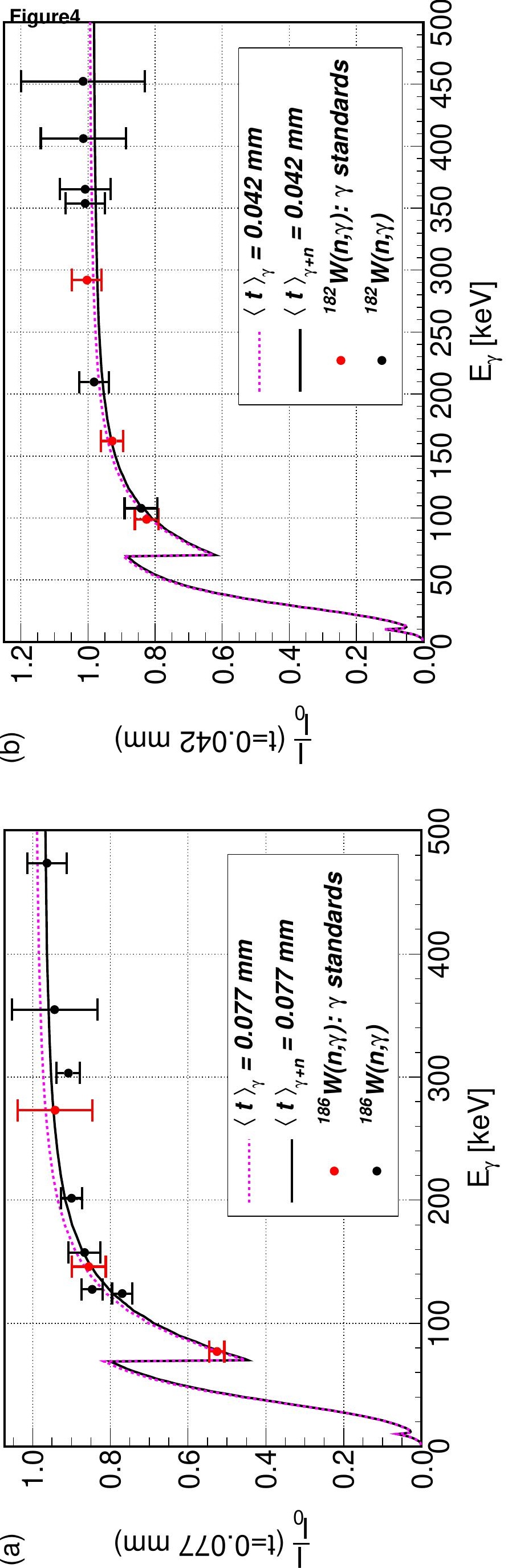


Click here to download LaTeX Source Files: nimb_xdx_r.tgz 